\title{
1 Ontology of RNA Sequencing (ORNASEQ) and its application
}

3 Stephen A Fisher

$4 \quad$ Biology Department

5 School of Arts and Sciences

6 University of Pennsylvania

$7 \quad 433$ S. University Avenue

8 Philadelphia, PA 19104

9 safisher@upenn.edu

10

11 Junhyong Kim*

12 Biology Department

13 School of Arts and Sciences

14 University of Pennsylvania

15433 S. University Avenue

16 Philadelphia, PA 19104

17 junhyong@upenn.edu

18

$19 *$ Corresponding author

20 


\section{Abstract}

22 Background: Next-generation RNA sequencing is a rapidly developing technology

23 with complex procedures encompassing different experimental modalities. As the

24 technology evolves and its use expand, so does the need to capture the data

25 provenance from these sequencing studies and the need to create new tools to

26 manage and manipulate these provenance stores.

28 Results: Here we used the Ontology for Biomedical Investigations (OBI) and many

29 other ontologies from the Open Biological and Biomedical Ontology (OBO) Foundry

30 as a framework from which to create an application ontology (ORNASEQ: Ontology

31 of RNA sequencing) to capture data provenance for next-generation RNA

32 sequencing studies. Additionally, we provide an extensive real-life sample

33 provenance data set for use in developing new provenance tools and additional

34 sequencing data models.

36 Conclusions: The Ontology of RNA Sequencing (ORNASEQ) provides core terms for

37 use in building data models to capture the provenance from next-generation RNA

38 sequencing studies. The supplied sample provenance data also exemplifies many of

39 the complexities of RNA sequencing studies and underscores the need for potent

40 workflow management systems.

\section{Keywords}

42 Ontology, RNAseq, PROV-XML 


\section{Background}

45 Until recently the cost of performing next-generation RNA sequencing (RNAseq)

46 experiments limited the amount of data generated by a single lab and managing and

47 properly documenting a few experiments was not fundamentally challenging.

48 However, as sequencing costs have dropped, research groups are now running

49 hundreds, thousands or even tens of thousands of RNAseq experiments, creating a

50 need to systematically document experimental and informatics details and track

51 provenance of the final published or publicly released datasets. RNAseq has also

52 begun making its way into medical diagnostics, where data provenance is a

53 necessity for quality assurance and regulatory compliance. Tracking the data

54 provenance for hundreds or thousands of sequencing experiments in either a

55 research or medical setting requires data models and structures that must be put

56 into place to capture the necessary information at all stages of a sequencing

57 experiment and it's not always obvious what information is necessary. While there

58 are numerous platforms and pipelines to analyze RNAseq data, there are limited

59 data models or ontologies that could be applied to successfully capture the details of

60 an RNAseq experiment [1-6].

62 Within a single next-generation sequencing experiment there is a dizzying amount

63 of information that must be captured throughout the often-complex experimental

64 procedures and post-sequencing informatics analyses. The problem is further 
65 complicated by the number of researchers or technicians who might be involved in a

66 single sequencing experiment whose roles are interspersed in irregular patterns;

67 complexity of biological specimens, their origin and experimental designs; and, the

68 frequent disconnect between the biologists running the experiments and the

69 bioinformaticists analyzing the data. Tracking data provenance spanning

70 experiment procedures recorded in lab notebooks belonging to multiple biologists

71 and computer log files residing in a series of cryptic directories on a file system

72 quickly becomes an intractable problem. These challenges suggest a need for a

73 comprehensive next generation sequencing provenance system. Data provenance

74 requires data models, provenance models, and supporting infrastructure. Here, we

75 focus on the first part of data models for RNA sequencing experiments and describe

76 an Ontology for RNA Sequences (ORNASEQ). In addition, we provide a large next-

77 generation sequencing use case from an active RNAseq workflow, using the PROV-

78 XML database format for the community to use as an example dataset for

79 development of provenance models and tools.

\section{Ontology for RNA sequencing}

81 The Ontology for RNA Sequencing (ORNASEQ) is an application ontology based

82 largely on the Ontology for Biomedical Investigations (OBI)[1], using the principles

83 of OBO Foundry[7]. Specifically, ORNASEQ contains 162 terms, 117 of the terms are

84 from 16 existing ontologies, with 28 new terms having now been added to OBI and

8517 terms being defined directly in ORNASEQ (see Table 1). ORNASEQ is designed to

86 annotate RNA-based next-generation sequencing, although much of ORNASEQ 
bioRxiv preprint doi: https://doi.org/10.1101/405720; this version posted August 31, 2018. The copyright holder for this preprint (which was not

certified by peer review) is the author/funder, who has granted bioRxiv a license to display the preprint in perpetuity. It is made available under aCC-BY 4.0 International license.

87 would also apply to DNA-based next-generation sequencing. The ontology was

88 designed, in part, through efforts to track the data provenance of thousands of

89 RNAseq samples collected by the NIH Common Fund Single Cell Analysis Program-

90 Transcriptome (SCAP-T) program[8]. Terms included in the ontology cover pre-

91 sequencing preparations and primary post-sequencing data analysis.

92 


\begin{tabular}{|c|c|}
\hline Ontology & Number Terms \\
\hline $\begin{array}{l}\text { BFO: Basic Formal Ontology } \\
\text { http://purl.obolibrary.org/obo/bfo.owl }\end{array}$ & 10 \\
\hline $\begin{array}{l}\text { CHEBI: Chemical Entities of Biological Interest } \\
\text { http://purl.obolibrary.org/obo/chebi.owl }\end{array}$ & 2 \\
\hline $\begin{array}{l}\text { CL: Cell Ontology } \\
\text { http://purl.obolibrary.org/obo/cl.owl }\end{array}$ & 6 \\
\hline $\begin{array}{l}\text { EFO: Experimental Factor Ontology } \\
\text { http://www.ebi.ac.uk/efo/efo.owl }\end{array}$ & 6 \\
\hline $\begin{array}{l}\text { GENEPIO: Genomic Epidemiology Ontology } \\
\text { http://purl.obolibrary.org/obo/genepio.owl }\end{array}$ & 4 \\
\hline $\begin{array}{l}\text { G0: Gene Ontology } \\
\text { http://purl.obolibrary.org/obo/go.owl }\end{array}$ & 5 \\
\hline $\begin{array}{l}\text { IAO: Information Artifact Ontology } \\
\text { http://purl.obolibrary.org/obo/iao.owl }\end{array}$ & 14 \\
\hline $\begin{array}{l}\text { NCBITaxon: NCBI Organismal Classification } \\
\text { http://purl.obolibrary.org/obo/ncbitaxon.owl }\end{array}$ & 1 \\
\hline $\begin{array}{l}\text { NCIT: NCI Thesaurus OBO Edition } \\
\text { http://purl.obolibrary.org/obo/ncit.owl }\end{array}$ & 6 \\
\hline $\begin{array}{l}\text { OBI: Ontology for Biomedical Investigations } \\
\text { http://purl.obolibrary.org/obo/obi.owl }\end{array}$ & 79 \\
\hline
\end{tabular}




\begin{tabular}{|c|c|}
\hline $\begin{array}{l}\text { OBIws: OBI web service, development version } \\
\text { http://purl.obolibrary.org/obo/obi/webService.owl }\end{array}$ & 5 \\
\hline $\begin{array}{l}\text { OGMS: Ontology for General Medical Science } \\
\text { http://purl.obolibrary.org/obo/ogms.owl }\end{array}$ & 1 \\
\hline $\begin{array}{l}\text { OMIABIS: Ontologized MIABIS } \\
\text { http://purl.obolibrary.org/obo/omiabis.owl }\end{array}$ & 2 \\
\hline $\begin{array}{l}\text { SO: Sequence Types and Features } \\
\text { http://purl.obolibrary.org/obo/so.owl }\end{array}$ & 1 \\
\hline $\begin{array}{l}\text { TAXRANK: Taxonomic rank vocabulary } \\
\text { http://purl.obolibrary.org/obo/taxrank.owl }\end{array}$ & 2 \\
\hline $\begin{array}{l}\text { UBERON: Uberon multi-species anatomy ontology } \\
\text { http://purl.obolibrary.org/obo/uberon.owl }\end{array}$ & 1 \\
\hline
\end{tabular}

93 Table 1: The set of external ontologies included in ORNASEQ.

\section{Pre-Sequencing Provenance}

96 Knowing what happens to a data sample prior to sequencing is essential to

97 understanding the analyzed data. The provenance surrounding the preparation of

98 sequencing data can prove invaluable to diagnosing aberrant results. These

99 protocols are often revised over time and as hardware and reagents evolve and a

100 multi-year study will likely include many versions of protocols with varying degrees

101 of differential change. Even in the controlled context of medical diagnostics, changes 
102 are inevitable, for example, when a reagent becomes discontinued or hardware are

103 upgraded.

104

105 When capturing experimental lab data provenance, it is difficult to know what to

106 capture and in what format (e.g. as fields in a database, as a Word or PDF document,

107 or even as a reference to an entry in an electronic notebook). As sequencing

108 preparation protocols are often distributed as PDF or Word documents, trying to

109 track changes across multiple such documents quickly becomes a tedious process

110 that is difficult to automate and nearly impossible to query for specific questions

111 (e.g., what version of sequencing chemistry was used for what samples). Conversely,

112 because the protocols involve many incremental steps and details that are inter-

113 related in a complicated manner, it is difficult to convert each protocol into a fine-

114 grained structured knowledge model suitable for standard DBMS. Similar to the

115 scheme implemented by the Genomic Standards Consortium [3], we propose

116 experimental provenance be captured in a hybrid fashion including both detailed

117 protocol files and hardcoded fields tracking fundamental datum external to the

118 protocol files. In this RNAseq data provenance schema, complete protocol

119 definitions are stored in the provenance database as files (typically PDF or Word

120 documents). Critical or commonly changed features of the protocol are additionally

121 captured in the database schema. While not optimal, this approach preserves the

122 fine detail required to replicate an experimental procedure, while allowing for

123 structured query of the main features. 


\section{Post-Sequencing Provenance}

126 When RNAseq data comes off the sequencer it is typically converted into fastq files

127 by a proprietary, sequencer-specific program. A fastq file is a text file containing

128 nucleotide sequences [9]. In the case of RNAseq, fastq files contain nucleotide

129 sequences representing the RNA molecules from a biological sample. Any one RNA

130 molecule might be represented by tens to hundreds of thousands of sometimes

131 duplicate nucleotide sequences in the fastq file, with each nucleotide sequence

132 referred to as a "read" (i.e. a read out of the biological sequence). The fastq files are

133 run through what is called a "primary analysis pipeline", which may include any

134 number of steps such as generating quality control metrics, removing contaminant

135 sequences from one or both ends of the reads, removing low quality sub-sequences

136 from reads, removing duplicate reads, and aligning reads to a pre-defined reference

137 library. Primary analysis pipelines and more generally RNA sequencing experiments

138 often culminate in the generation of gene-, transcript-, or exonic-counts of the

139 number of reads associated with each of these categories. The various steps will

140 mostly remain consistent within a research group, a project or a medical diagnostic

141 test. However, programs evolve, algorithmic bugs are fixed, and reference libraries

142 are refined. As with wet lab procedures, it is common and often necessary for

143 primary analysis pipelines to change by varying degrees over time. Since the data

144 and processes for the informatics steps are already machine readable, capturing

145 provenance for RNAseq analysis pipeline is a more obvious task than in a wet lab

146 context. However, relevant provenance information is typically realized as a set of

$147 \log$ files spread across a series of programs, each with specific directory structures. 
148 Using log files to track provenance data also quickly breaks down as programs

149 change or pipelines evolve and often requires complex programming to perform

150 simple queries across data samples. As with tracking wet lab data, provenance from

151 sample processing pipelines needs to be rigorously captured and systematically

152 stored in a central database. Addressing these challenges require incorporation of a

153 well-defined and use-case oriented ontology for the provenance objects, which we

154 provide with ORNASEQ.

\section{Sample Data}

156 ORNASEQ is meant to provide core terms used to track the provenance of RNAseq

157 datasets. However, any particular experiment or RNAseq use case will require a

158 multitude of additional terms. Here we provide a dataset containing curated and

159 modified data provenance from 1,347 next-generation sequencing samples. The

160 dataset contains 93 data fields, using ORNASEQ terms as appropriate. Each sample

161 was processed with one of four different versions of a primary analysis pipeline and

162 there was from one to three variants (sub-versions) of each pipeline version.

163 Specifically, samples were processed either as "single-end" or "paired-end" and

164 aligned with either STAR[10] or Bowtie[11, 12], ultimately leading to nine possible

165 pipeline variants. The dataset is provided as PROV-XML (see Additional file 2), with

166 the data summarized in an Excel table (see Additional file 1). Real world use cases

167 usually include messy data. Occasionally pipelines are run incorrectly either

168 through intentional operator actions or error. It's also quite common to have

169 missing or incomplete data provenance again through user actions (e.g. data was 
170 pulled from a source that lacked sufficient provenance), programming errors,

171 computer issues, etc. The dataset provided here intentionally includes both

172 incorrect and missing data.

173

$174 \quad$ Primary Analysis Pipeline Stages

175 The data provided describes an analysis pipeline with seven possible stages but

176 with each analysis only including five of the seven stages. Table 2 includes a subset

177 of data tracked for each pipeline stage and which stages might be used in a

178 particular pipeline version. For example, the stage HTSeq was only used in pipeline

179 version 1.0 while VERSE was only used in later pipeline versions. The stages are

180 very briefly described here and more complete descriptions can be found in the

181 PennSCAP-T Pipeline[13].

182 - BLAST - a subset of samples was aligned to the Blast NR database,

183 using BLAST as a quality control check.

$184 \quad \circ$ FastQC - FastQC is used as an additional quality control check.

$185 \quad \circ$ TRIM - contaminant sequences were removed from reads.

$186 \quad \circ$ BOWTIE - Bowtie was used to align reads to a reference genome.

$187 \quad \circ$ STAR - STAR was used to align reads to a reference genome.

$188 \quad \circ$ HTseq - HTSeq was used to assign aligned reads to genes.

189

- VERSE - VERSE was used to assign aligned reads to genes.

190

191 In the accompanying dataset, samples were aligned with either Bowtie or STAR but

192 not both. Similarly, alignments were processed by either HTSeq or VERSE, but not 
bioRxiv preprint doi: https://doi.org/10.1101/405720; this version posted August 31, 2018. The copyright holder for this preprint (which was not certified by peer review) is the author/funder, who has granted bioRxiv a license to display the preprint in perpetuity. It is made available under aCC-BY 4.0 International license.

193 both. The Excel table highlights which provenance terms are consistent within a

194 pipeline version. For example “BLAST.blastn.version” has pipeline version-specific

195 values. Provenance terms that might contain incorrect values are also denoted. For

196 example, the value for "STAR.star.version" in Pipeline version 2.0.1 might be

197 missing.

198 


\begin{tabular}{|c|c|c|c|c|c|}
\hline STAGE & PARAMETER & \multicolumn{4}{|c|}{ VALUE } \\
\hline \multirow{2}{*}{ BLAST } & blastn version & 2.2 .25 & 2.2 .30 & 2.2 .30 & 2.2 .30 \\
\hline & parseBlast.py version & 1.1 & 1.5 & 1.5 & 1.8 \\
\hline FASTQC & fastqc version & 0.10 .1 & 0.11 .2 & 0.11 .2 & 0.11 .2 \\
\hline \multirow{5}{*}{ TRIM } & trimReads.py version & 0.6 & 0.6 & 0.6 & 0.7 .2 \\
\hline & removeN & 1 & 1 & 1 & 1 \\
\hline & minLen & 30 & 20 & 20 & 20 \\
\hline & phredThresh & - & 53 & 53 & 53 \\
\hline & numAT & 30 & 26 & 26 & 26 \\
\hline \multirow{3}{*}{ BOWTIE } & bowtie version & 0.12 .7 & 1.1 .1 & & 2.2 .9 \\
\hline & minins & 250 & 150 & & 150 \\
\hline & maxins & 450 & 600 & & 600 \\
\hline \multirow{2}{*}{ STAR } & star version & 2.3.0.1 & 2.4.0h1 & $2.4 .0 \mathrm{j}$ & $2.5 .1 b$ \\
\hline & samtools version & 1.1 & 1.1 & 1.2 & 1.2 \\
\hline HTSEQ & htseq version & $0.5 .4 \mathrm{p} 5$ & & & \\
\hline \multirow[t]{3}{*}{ VERSE } & verse version & & $\mathrm{v} 0.1 .4$ & $\mathrm{v} 0.1 .4$ & $\mathrm{v} 0.1 .5$ \\
\hline & Pipeline Version & 1.0 & 2.0 & 2.0 .1 & 2.1 \\
\hline & Number Samples & 208 & 413 & 216 & 510 \\
\hline
\end{tabular}

199 Table 2: Subset of pipeline-specific parameters included in sample dataset, across

200 pipeline stages and pipeline versions.

\section{Conclusion}


202 Next generation sequencing (NGS) is a complex technology with multiple varied

203 steps. Capturing the provenance of NGS data requires complex systems and multi-

204 user collaborations. The schemas and ontology we define here offer a basic

205 framework that can be tuned and expended by researchers to the particulars of

206 individual studies, while providing basic commonalities across studies.

207

208 The dataset provided illustrates some of the complexities of the data provenance

209 from downstream processing of NGS data. These complexities will grow as the field

210 evolves. For example, there are now hundreds of variants of basic sequencing

211 protocol that are specific to particular biology applications (e.g., ATAC-seq; [14];

212 Drop-Seq; $[15,16])$. Each of these involve variations in experimental and informatics

213 processes. It will become necessary to build workflow management systems and

214 smart clustering algorithms of the provenance[17] to help segment incomplete and

215 erroneous data. We propose that our large real-life dataset example will prove

216 useful in designing future new workflow systems and provenance models.

\section{List of Abbreviations}

218 OBI: Ontology for Biomedical Investigations

219 ORNASEQ: Ontology of RNA Sequencing

220 OBO: Open Biological and Biomedical Ontology

221 RNAseq: next-generation RNA sequencing

222 SCAP-T: NIH Common Fund Single Cell Analysis Program-Transcriptome

223 NGS: Next generation sequencing 


\section{Declarations}

225 Ethics approval and consent to participate

226 not applicable

227 Consent for publication

228 not applicable

229 Availability of data and material

230 The ontology presented in the current study is available from GitHub,

231 http://doi.org/10.5281/zenodo.1311869. The sample dataset presented in the

232 current study is available as additional article files.

233 Competing interests

234 The authors declare that they have no competing interests.

235 Funding

236 This work was supported in part by NIH grants 5U01EB020954 and U01MH098953.

237 The NIH played no role in the results of this research effort or the text of this paper.

238 Authors' contributions

239 SF and JK contributed to the design of this study and preparation of the manuscript.

240 Acknowledgements

241 We are grateful to Christian Stoeckert and Daniel Berrios for valuable feedback and

242 assistance adding terms to the Ontology for Biomedical Investigations. 


\section{References}

244 1. Bandrowski A, Brinkman R, Brochhausen M, Brush MH, Bug B, Chibucos MC, et al.

245 The Ontology for Biomedical Investigations. PLoS One. 2016;11:e0154556.

246 doi:10.1371/journal.pone.0154556.

247 2. Society FGD. Minimum Information about a high-throughput SEQuencing

248 Experiment. http://fged.org/projects/minseqe/.

249 3. Yilmaz P, Kottmann R, Field D, Knight R, Cole JR, Amaral-Zettler L, et al. Minimum

250 information about a marker gene sequence (MIMARKS) and minimum information

251 about any (x) sequence (MIXS) specifications. Nat Biotechnol. 2011;29:415-20.

252 doi:10.1038/nbt.1823.

253 4. Sansone SA, Rocca-Serra P, Field D, Maguire E, Taylor C, Hofmann O, et al. Toward

254 interoperable bioscience data. Nat Genet. 2012;44:121-6. doi:10.1038/ng.1054.

255 5. Global Alliance for Genomics \& Health. 2018. https://www.ga4gh.org.

256 6. Hong EL, Sloan CA, Chan ET, Davidson JM, Malladi VS, Strattan JS, et al. Principles

257 of metadata organization at the ENCODE data coordination center. Database

258 (Oxford). 2016;2016. doi:10.1093/database/baw001.

259 7. Smith B, Ashburner M, Rosse C, Bard J, Bug W, Ceusters W, et al. The OBO

260 Foundry: coordinated evolution of ontologies to support biomedical data

261 integration. Nat Biotechnol. 2007;25:1251-5. doi:10.1038/nbt1346.

262 8. Single Cell Analysis Program - Transcriptome Project. http://scap-t.org/.

263 9. Cock PJ, Fields CJ, Goto N, Heuer ML, Rice PM. The Sanger FASTQ file format for 
264 sequences with quality scores, and the Solexa/Illumina FASTQ variants. Nucleic

265 Acids Res. 2010;38:1767-71. doi:10.1093/nar/gkp1137.

266 10. Dobin A, Davis C a, Schlesinger F, Drenkow J, Zaleski C, Jha S, et al. STAR:

267 ultrafast universal RNA-seq aligner. Bioinformatics. 2013;29:15-21.

268 doi:10.1093/bioinformatics/bts635.

269 11. Langmead B, Trapnell C, Pop M, Salzberg SL. Ultrafast and memory-efficient

270 alignment of short DNA sequences to the human genome. Genome Biol.

271 2009;10:R25. doi:10.1186/gb-2009-10-3-r25.

272 12. Langmead B, Salzberg SL. Fast gapped-read alignment with Bowtie 2. Nat

273 Methods. 2012;9:357-9. doi:10.1038/nmeth.1923.

274 13. Fisher J. SA. K. PennSCAP-T Pipeline. 2018. https://github.com/safisher/ngs.

275 14. Buenrostro JD, Wu B, Chang HY, Greenleaf WJ. ATAC-seq: A Method for Assaying

276 Chromatin Accessibility Genome-Wide. Curr Protoc Mol Biol. 2015;109:21 29 1-9.

277 doi:10.1002/0471142727.mb2129s109.

278 15. Klein AM, Mazutis L, Akartuna I, Tallapragada N, Veres A, Li V, et al. Droplet

279 barcoding for single-cell transcriptomics applied to embryonic stem cells. Cell.

$280 \quad$ 2015;161:1187-201. doi:10.1016/j.cell.2015.04.044.

281 16. Macosko EZ, Basu A, Satija R, Nemesh J, Shekhar K, Goldman M, et al. Highly

282 Parallel Genome-wide Expression Profiling of Individual Cells Using Nanoliter

283 Droplets. Cell. 2015;161:1202-14. doi:10.1016/j.cell.2015.05.002.

284 17. Alawini L.; Davidson, S.; Fisher, S.; Kim, J. A. C. Discovering Similar Workflows via 
285 Provenance Clustering: a Case Study. In: Belhajjame K, Gehani A, Alper P, editors.

286 Provenance and Annotation of Data and Processes. London: Springer International

287 Publishing; 2018.

288

289 Additional Files

290 Additional file 1

291 Format: Excel Workbook from Excel for Mac version 16 (.xlsx)

292 Title: A Large Curated RNAseq Metadata Dataset

293 Description: This file contains a curated dataset consisting of metadata collected

294 from 1347 next-generation sequencing samples. The file also contains a set of terms

295 that can be used to separate the data samples between nine primary analysis

296 pipelines.

297 Additional file 2

298 Format: XML file (.xml)

299 Title: PROV-XML Instantiation of a RNAseq Metadata Dataset

300 Description: This is an XML file that contains a PROV-XML representation of the

301 data included in "Additional file 1.xslx"; that is, an example dataset containing

302 metadata from 1347 RNAseq data samples that were processed with one of nine

303 primary analysis pipelines. 